\title{
An Analysis of the Musical Writing Features of Chopin's Scherzo No. 2 in B-flat minor
}

\author{
Yu Liu \\ Xi’an Shiyou University, Xi’an Shaanxi, 710065, China
}

Keywords: Chopin, Harmonic music, Creative characteristics.

\begin{abstract}
Scherzo No. 2 in B-flat minor is the most famous one of Chopin's four piano harmonies. This article analyzes and summarizes the important piano works by Scherzo No. 2 in B-flat minor. Each part of the music image, musical composition and musical characteristics elaborates the musical composition of Scherzo No. 2 in B-flat minor.
\end{abstract}

\section{Introduction}

Chopin Scherzo No. 2 in B-flat minor was published in December 1837 and entitled "The Duchess of Forststein." During this period, Chopin was in the full period of artistic creation. Therefore, the work was beautifully melodious, passionate and passionate, and the mood of the music was slow and urgent. There was no tragic factor at all and it showed a very high artistic achievement. The song also became Chopin IV. The most famous one in the first twist.

In the sonatas and symphonies, Beethoven used harpsichords instead of mini-steps, which laid a strong brush on the development of harbuterism. After inheriting the creative skills of Haydn, Mozart and Beethoven, Chopin has greatly developed this genre. Chopin's treacherous music is large in scale, profound in content, complex in structure, and rich in change. It is not limited to displaying witty and humorous styles. The combination of diverse melody and complex and varied character traits has transformed Chopin's scherzo into a new one, almost becoming a A new kind of music genre. Chopin's unique music creation style has been perfectly embodied in Scherzo No. 2 in B-flat minor, including the following aspects.

\section{Patriotism and national music elements throughout the music creation}

Chopin always believed that in addition to expressing the theme of the times, art should also show a distinct nationality. Scherzo No. 2 in B-flat minor The full melody reflects Chopin's deep patriotism from all aspects including melody, tone, texture, and harmony. For example, the Polish folk song is a monophonic melody. The melody of sincerity, enthusiasm, and softness are artistically appealing. The subject of the vice department of the Chopin No. 2 in B-flat minor exhibition department is obviously influenced by the Polish folk songs, and the melody is full of strong. Strong Polish style. (Spectrum Example 1) Chopin also inherited the repetition of homonyms in Polish folk songs, but only made a wide change in rhythm. These Polish-influenced musical elements cherish the memory of the musicians' deep remembrance of the motherland, and have also given rise to the theme of Chopin's Scherzo No. 2 in B-flat minor.

Sample 1 77-97 


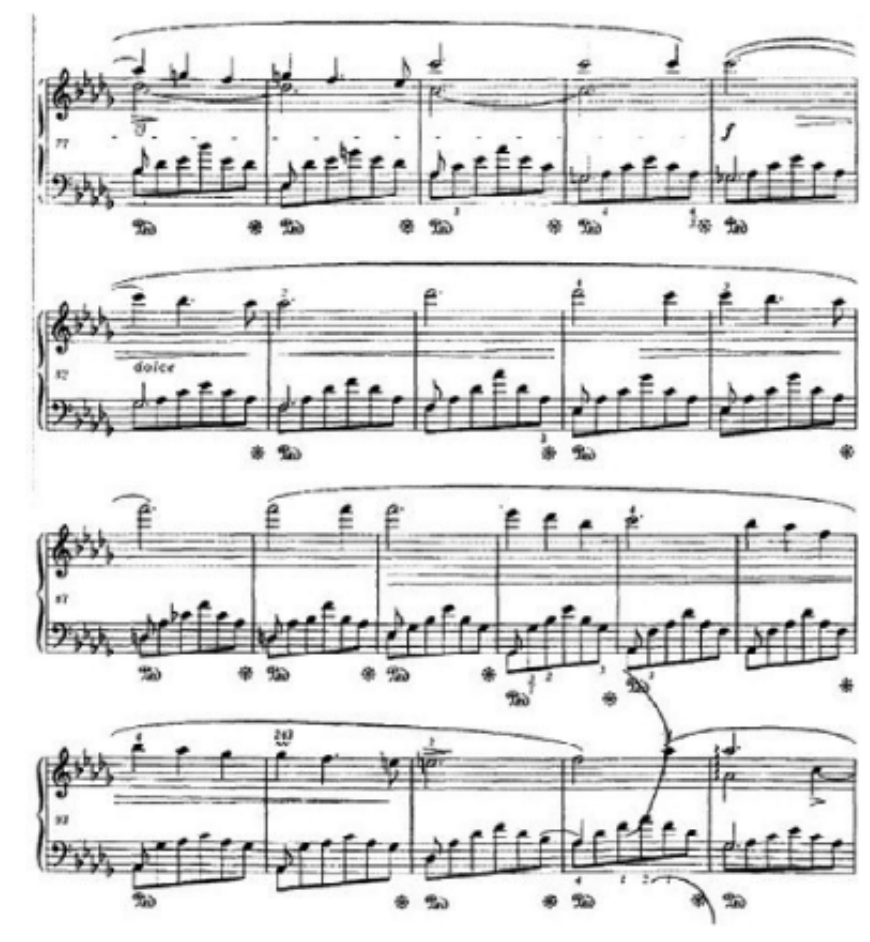

\section{Melody characteristics of melody and instrumental music}

Influenced by Italian opera and Polish folk songs, Chopin used a large number of decorative sounds in the melody, which greatly enriched the musical expression of the work. Under the embellishment of these small musical notes, the melody lines of the works are more gorgeous, and the flower-like music language sets the theme romantic and charming, so that people can not ignore the important role of these decorative sounds.

Sample 2 265-284
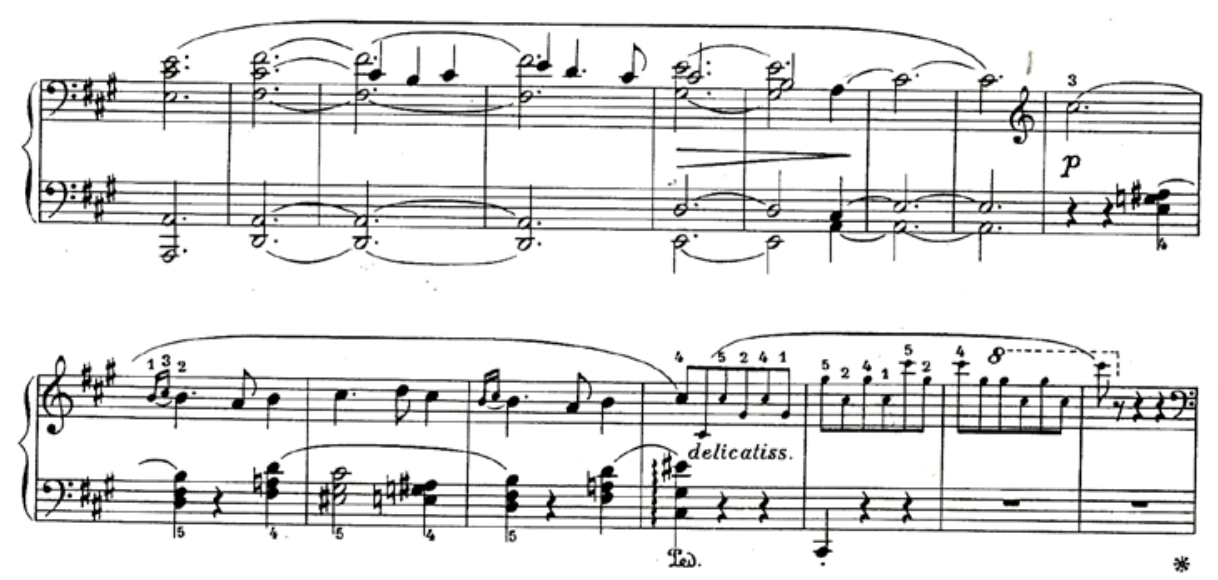

In Scherzo No. 2 in B-flat minor, Chopin also creatively applied the musical elements of the Italian opera's repertoire to the creation of a scherzo, giving people a fresh look. The two different themes seem to be talking softly to each other, and seemingly reciting poems in affectionate words, leaving a lasting impression on me. For example, the second theme of the Scherzo No. 2 in B-flat minor is the four-part texture of the composition method of composer home polyphony. In the second theme, the tune of the upper part is a waltz style, and the middle part is light and slightly uneasy. The downside of the triplet sounds like a girl's sigh. The two parts form an interesting contrast, as if two characters were on the stage. Talking softly greatly enhanced the lyricism and vocality of the melody. (Sample 3)

Sample 3 310-323 

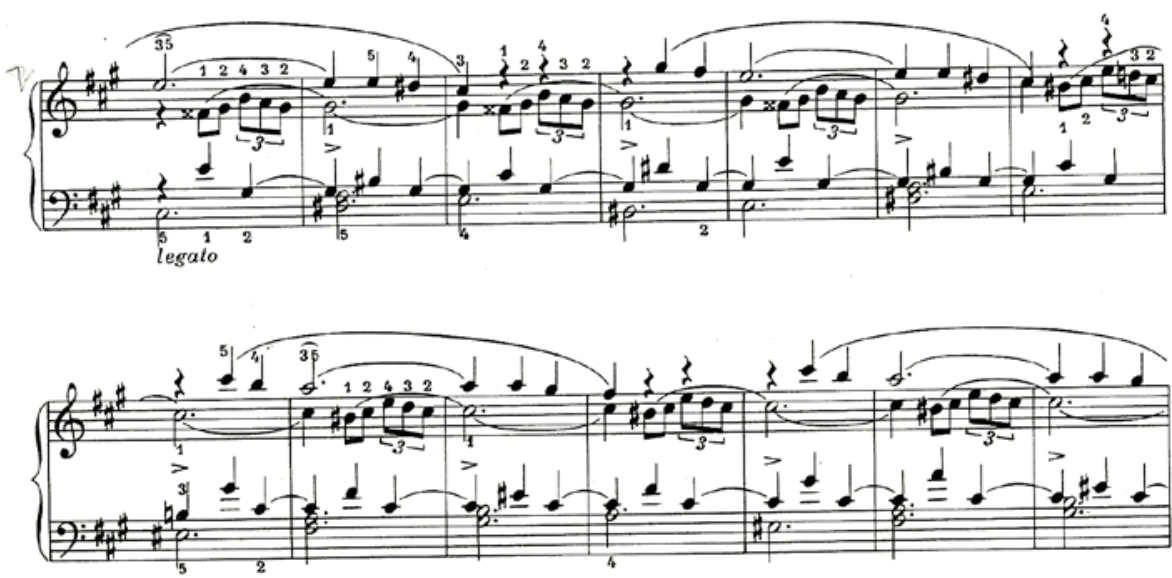

In Chopin's Scherzo No. 2 in B-flat minor, the skilled combination uses vocal melody and instrumental melody, and the instrumental melody and melody melody merge with each other, making the melody of the work colorful, romantic and moving. Enhanced jazz music expression. For example, in the third part of the music, a vocality treble set against the instrumental accompaniment sound pattern repeatedly appears, and the tunes are noble and elegant, which expresses the author's far-reaching and profound artistic conception. (See Spectrum Sample 4)

In Chopin's writing, vocal melody and instrumental melody are sometimes not so clear, the author consciously transforms the two into each other, when the melody with vocal characteristics gradually transforms into instrumental melody, or the instrumental melody transforms into vocal in the next phrase When it comes to sexual melody, it creates a dramatic and dramatic effect that greatly enhances the musical expression of the work. Obviously, the transformation between the two melodies is even more difficult. This is one of the reasons that Chopin's contemporary composers rarely attempted this technique. Chopin's skillful use and control of the changes between vocalized melody and instrumental melody, demonstrated composer's creative skills. For example, the latter half of the first theme of the music development department began as a melodious melodious melody, with a melodious and harmonious mood under the embellishment of delicate decoration, and the sentence endings changed gradually from high to low. The decomposition chord type, which is obviously a melody with instrumental characteristics. The melody melody and instrumental melody have appeared one after another, giving people a very deep impression. (See Sample 2)

Sample 4 52-80
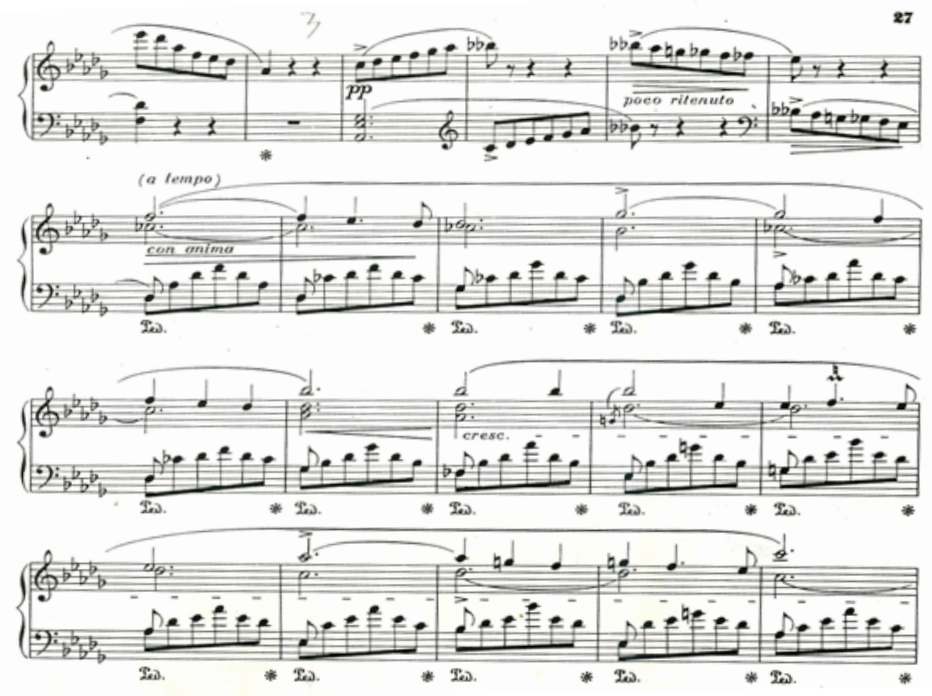

\section{Bold revolutionary harmonic and tonal applications}

In the 19th century, classical music entered the romantic period. At this stage, the composer pays more attention to his own subjective feelings and expresses his personal emotions. Composers in the classical period often strictly followed the requirements of traditional harmony techniques and 
pursued the harmony and regularity of harmony. Chopin, like musicians of the same period, prefers semitone harmonization, non-functional harmony, and color harmony. He widely uses complex harmonics such as discordant chords and chords. The bold, multi-colored romanticism and vocals make the audience refreshed. As a great reformer of the Romantic period and acoustics, Chopin has created many new techniques in the use of harmony. His harmonies not only widely absorbed classical music traditions, but also absorbed nutrition from folk music. Chopard's attainment of the harmonization realm has left other composers in the same period far behind. Rimsky Korsakov praised Chopin as "the most talented and unique harmony artist" and "has two extraordinary talents: the talents of the greatest melody and the most talented and unique. Sonata's talents." In the composer's creation, he not only breaks the convention in the genre of music, but also promotes the harmony in terms of harmony and dares to try new techniques. In Scherzo No. 2 in B-flat minor, harmony effects are complex, creative harmonic vocabulary and unexpected transitions create highly expressive harmony.

Chopin is adept at using sophisticated harmonics to create complex and varied sound effects. The use of a large number of chords and sounds combined with simple chords to promote the gradual advancement of the music structure, the harmony effect is perfect. In the first topic of Scherzo No. 2 in B-flat minor, the simple harmony gave a fresh look and caught the listener's heart. Using only the two triads I and IV, the use of longer harmonic rhythms and a large number of extra-vocal sounds increases the feeling of inharmony, revealing the composer's informal, emotionally rich inner world. (Sample 5)

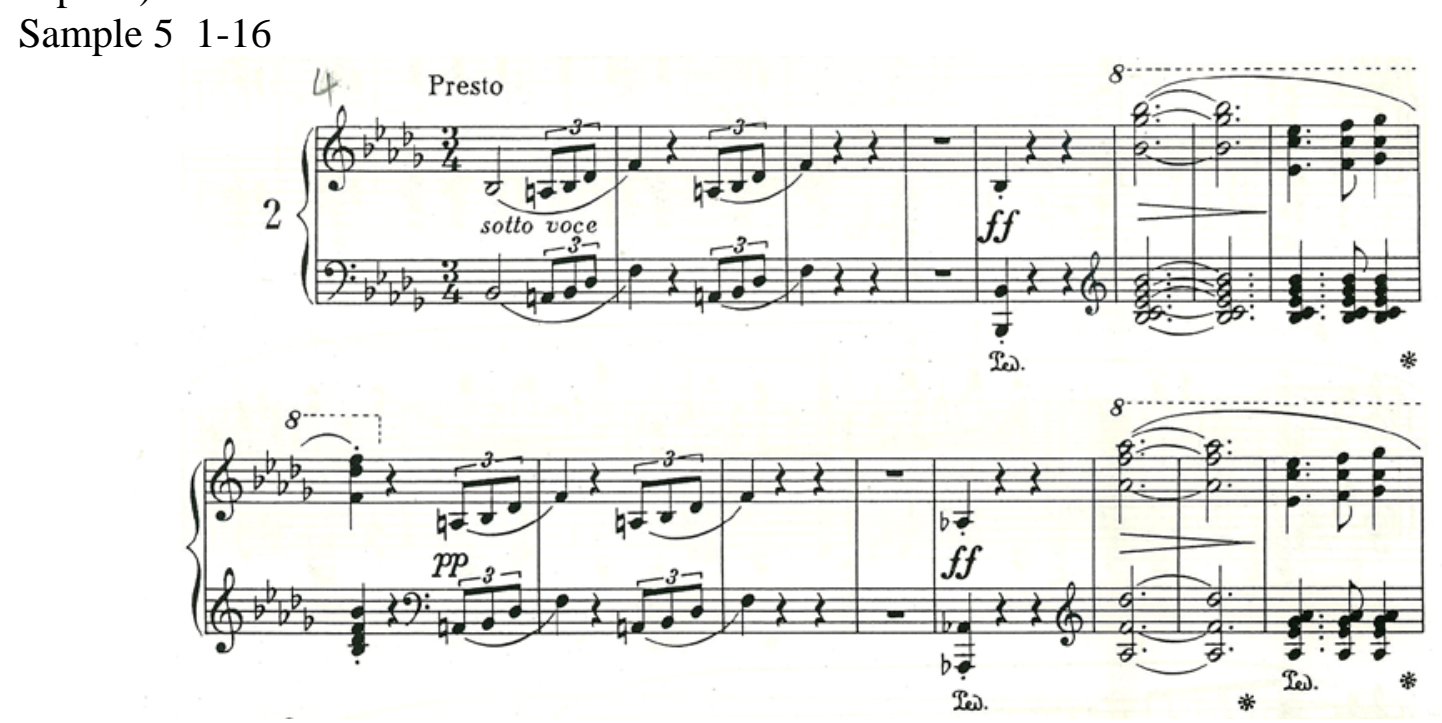

Chopin has boldly reformed traditional harpsichords in the use of tonality, and has "freely explored the relationship between tone and contrast in the tonality and additional colors." In Scherzo No. 2 in B-flat minor, the music begins in b flat minor, but it ends in D flat major. Two completely different modes do not have a master/slave distinction, but blend with each other but show up independently. The composer's jaw-dropping ability to innovate and use sophisticated techniques.

The third degree juxtaposition is one of the composers often used in the Romantic period, that is, to repeat the same melody repeatedly in order to emphasize the juxtaposition. Chopin often emphasizes the expressiveness of the harmony color by means of three degrees of juxtaposition. In Scherzo No. 2 in B-flat minor, Chopin intentionally created a typical three-degree juxtaposition on several subjects in the middle segment: from A major to $C$ minor and then to E major. (Sample 6)

Sample 6 265-284; 310-324; 334-344 

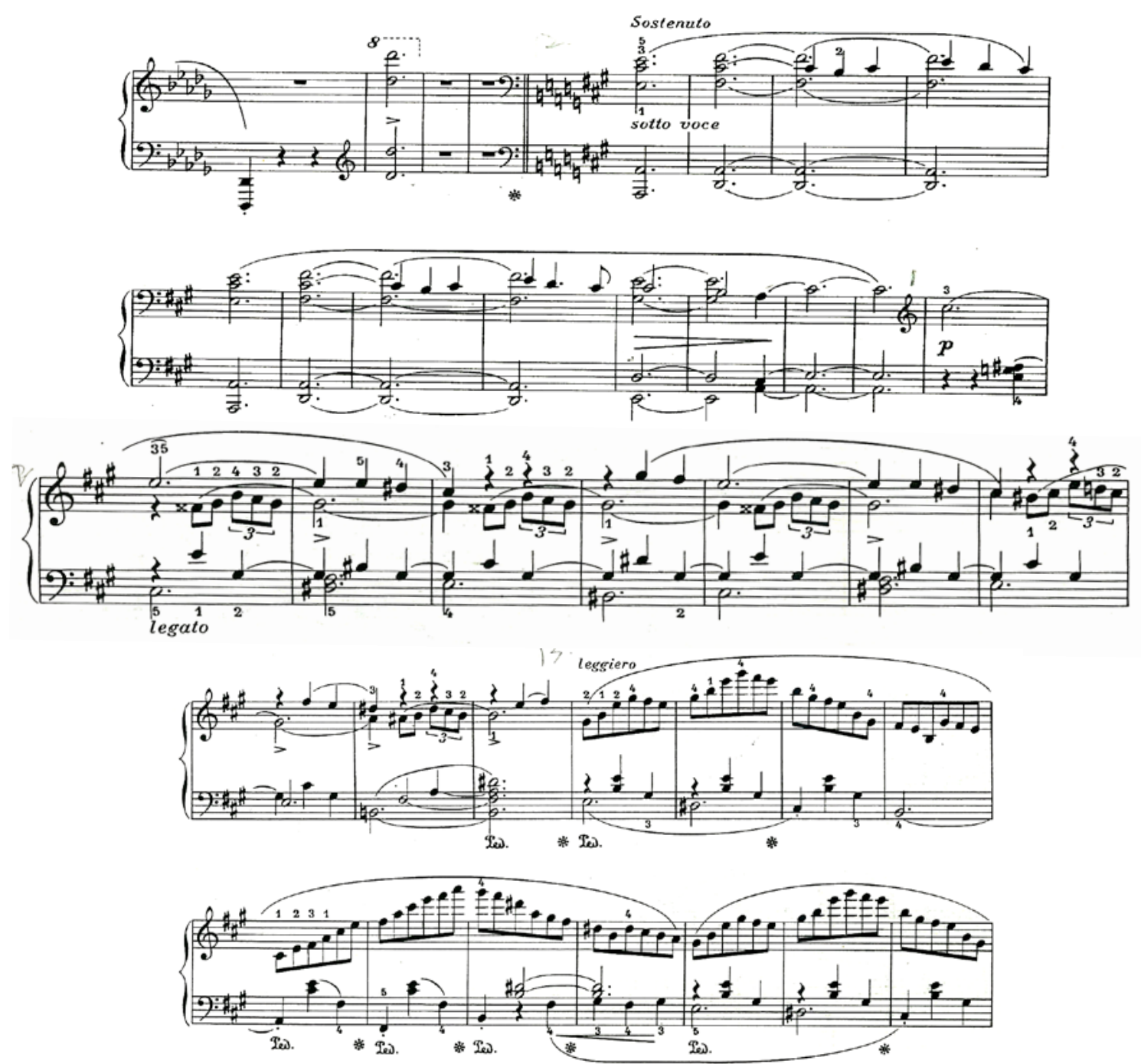

In his works, Chopin also boldly uses alternation styles and sudden and sudden transfer to change harmony, creating a dramatic and intense dramatic musical effect. Chopin also repeated the same melody fragment in different tones to enhance the romanticism of the work. As shown in the figure below, the composer uses three phrases to repeat the same melody in D major, E major, and A major, respectively, with distinctive Polish folk music characteristics. (Sample 7)

Sample 7 49-64; 517-519; 816-819
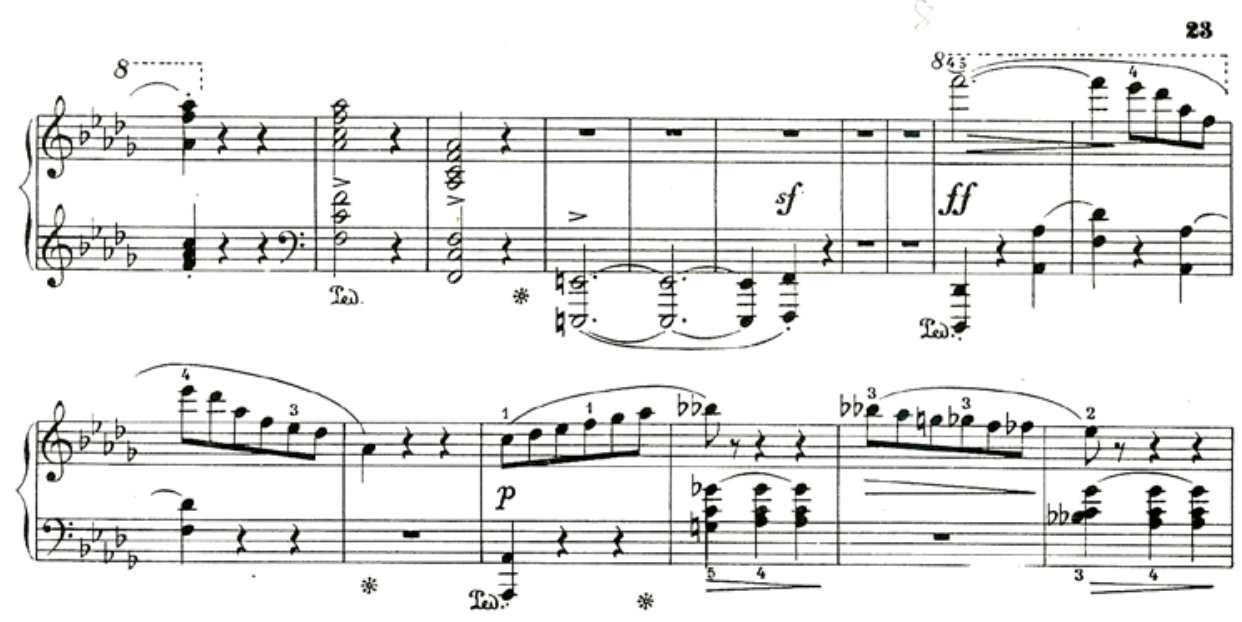

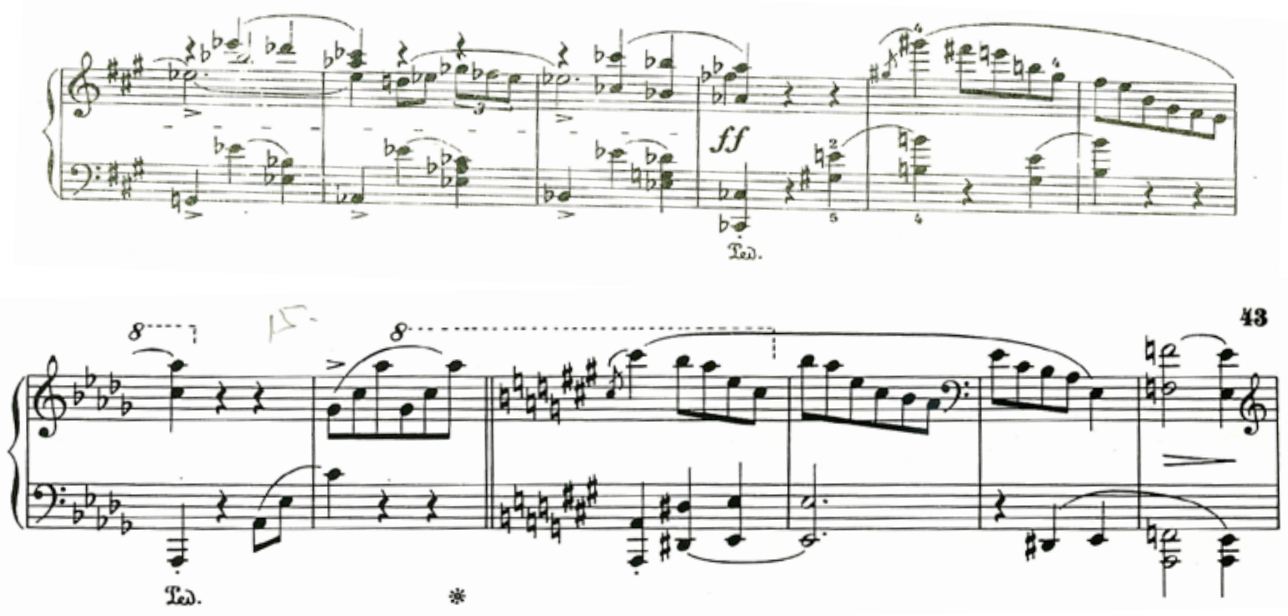

Chopin's great achievements in harmony made him unique in the music field in the early 19th century. Scherzo No. 2 in B-flat minor has bold and harmonious language and rich artistic expression. He is still loved by music lovers.

\section{A distinctive, rich and varied piano texture}

Chopin is recognized as the "Master of Piano Orchestration" and the distinctive and varied piano texture in Scherzo No. 2 in B-flat minor proves to the world once again that piano music is a "voice language." Skillful texture writing The technique shows Chopin's impressive musical talent at the peak of his creation.

Chopin attaches great importance to Fugue's genre. He systematically studied Bach's polyphonic works and drew nutrition from the work of classical composers. However, Chopin is not confined to the structure of traditional polyphonic textures, but instead integrates the voices organically under the premise of ensuring the melody lines of each voice. In the middle part of Scherzo No. 2 in B-flat minor, Chopin organically combines the four parts, the right-hand treble part sings alone, the sub-high part replenishes the right-hand treble part, and the left-hand part has its own part. The main melody echoes with the right-hand voice. The four voices blend with each other to form a beautiful and harmonious whole. (See Spectrum Example 2)

Chopin attaches great importance to piano accompaniment and sometimes even forgets to eat better accompaniment music. Therefore, the accompaniment texture in Chopin's works has exceeded the general structure of the accompaniment of contemporary piano music and even surpasses the melody subject. In the first part of Scherzo No. 2 in B-flat minor, the piano texture in the lyrical passage uses decomposed chords, renders the soft and delicate background sound through the pedals, and the fluid music structure combines with the musical theme to create a charm Sound screen. (See Sample 4)

\section{Large and complex musical structure}

Chopin inherited and developed 18th century harbuteristic creation traditions. Based on the traditional form structure, the harpsichord music was separated from the large set of musical compositions, giving the harbuterious music genre a new life. The adoption of the hybrid musical structure and the richness of the creative content make the ambiguous musical structure increasingly large and complex.

Scherzo No. 2 in B-flat minor adds a small amount of swirling and variation factors to the sonata structure, and the melody development of the composition of the music shows that the main part, the connecting part, and the secondary part emphasize the main part again. The independent middle paragraph uses the principle of variation to reflect the change of the theme, and the difference between the tonality in the reproduction department and the presentation department, once again creates a dramatic tension and promotes the continuous development of the music. (Sample 8) 
Sample 8 691-707
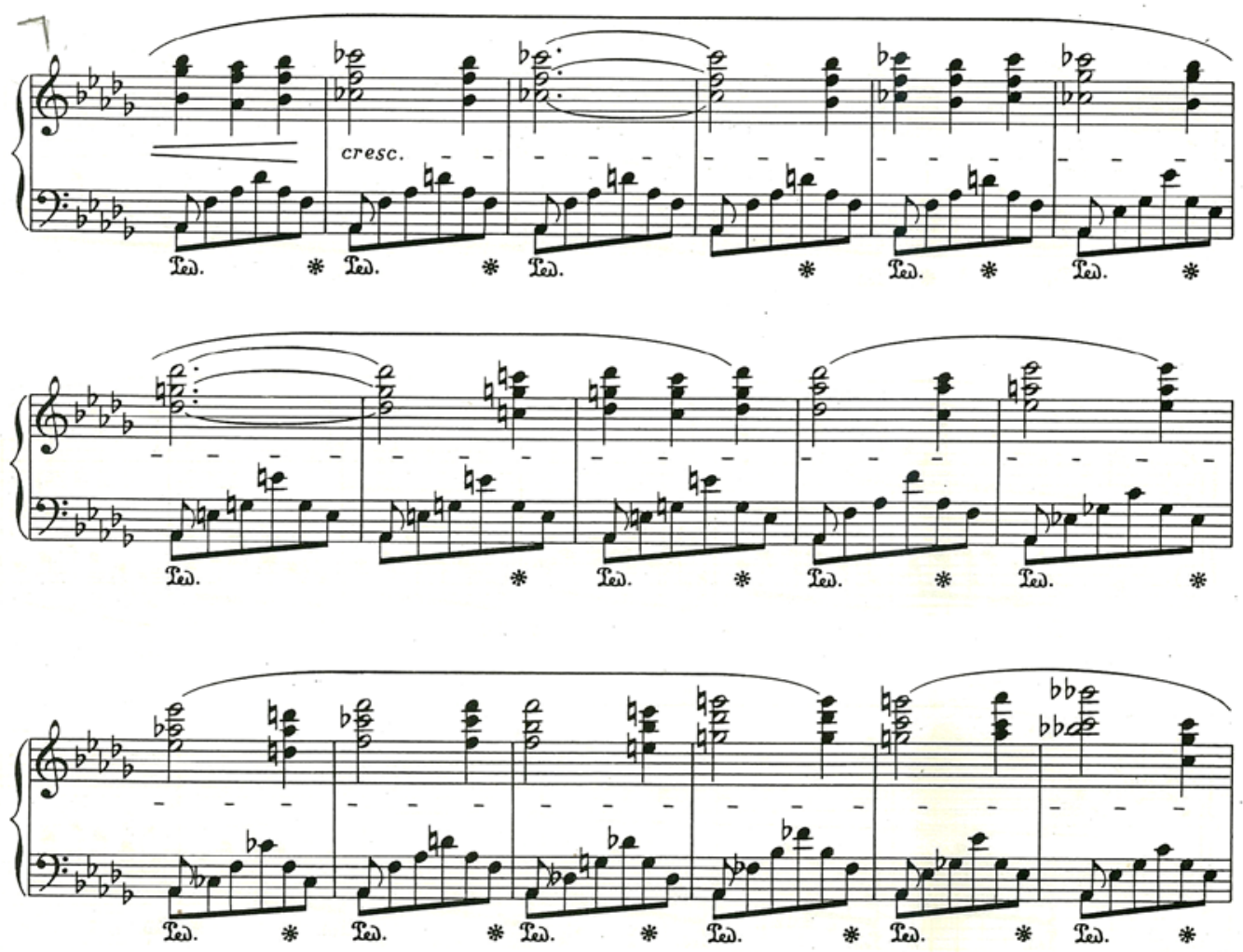

As one of the most poetic works of Chopin, the "poet", Schumann once commented, "This humbling enthusiasm character is reminiscent of the previous Chopin's composer, and it is very bold, simple, easy, bold and cute. The odious and evil are all melted down in a furnace. It is a highly conceived piece of music that is not inferior to any of Sir Pooh's poetry." Chopin's scherzo is no longer limited to witty, humorous style limitations, profound Thoughtful and dramatic creative techniques, shocking passion and imaginary musical image made Chopin greatly surpass other contemporary musicians and left memorable music memories for people.

\section{References}

[1] Liu Jinqing. The Artistic Characteristics of Lyric Melody in Chopin's Piano Works, Huang Zhong, Journal of Wuhan Conservatory of Music, 2000,04:68-73.

[2] Li Wei. Music Analysis and Performance Tips of Chopin Scherzo No. 2 in B-flat minor. Symphony. 1996,01, 29-31.

[3] A. Solothoff. Chopin's creation, Beijing: People's Music Publishing House, 2005.

[4] Zhu Yafeng. Chopin's Romanticism, Piano Art, 2000(1).

[5] Wang Xiaoke. On the Musical Features of Chopin's Harmony, Music research, 2009(4).

[6] Zhu Xiaoju. Chopin Harmonic Studies, Shanghai Conservatory of Music, 2006(6).

[7] Hu Yuqing, Liu Jia. On the National Characteristics of Chopin's Music, Music exploration, 2000(3). 